\title{
Rapid, Experience-Dependent Changes in Levels of Synaptic Zinc in Primary Somatosensory Cortex of the Adult Mouse
}

\author{
Craig E. Brown and Richard H. Dyck \\ Department of Psychology, University of Calgary, Calgary, Alberta, Canada T2N IN4
}

Electrophysiological studies have established that the adult cerebral cortex undergoes immediate functional reorganizations after perturbations of the sensory periphery. These activity-dependent modifications are thought to be mediated via the rapid regulation of the synaptic strength of existing connections. Recent studies have implicated synaptic zinc as contributing to activity-dependent mechanisms of cortical plasticity, such as long-term potentiation and long-term depression, by virtue of its potent ability to modulate glutamatergic neurotransmission. To investigate the role of synaptic zinc in cortical plasticity, we examined changes in the barrel-specific distribution of zinc in axon terminals innervating the primary somatosensory cortex of adult mice at different time points after whisker plucking. In layer IV of normal adult mice, zinc staining in the barrel field was characterized by intense staining in inter-barrel septae and low levels of staining in barrel hollows. Within $3 \mathrm{hr}$, and up to 1 week after the removal of a row of whiskers, zinc staining increased significantly in barrel hollows corresponding to the plucked whiskers. With longer survival times, levels of zinc staining gradually declined in deprived barrel hollows, returning to normal levels by 2-3 weeks after whisker removal. Increased levels of zinc staining in deprived barrel hollows were highly, negatively correlated with the length of whiskers as they regrew. These results indicate that levels of synaptic zinc in the neocortex are rapidly regulated by changes in sensory experience and suggest that zinc may participate in the plastic changes that normally occur in the cortex on a moment-tomoment basis.

Key words: zinc; experience-dependent plasticity; somatosensory cortex; whiskers; adult; mouse
The synaptic organization of the adult cerebral cortex is continuously modified by sensory experience. In the visual cortex, electrophysiological studies have demonstrated that manipulations of visual experience produce immediate reorganizations in receptive field size and cortical topography (Gilbert and Wiesel, 1992; Trachtenberg et al., 2000). Comparably, digit amputation in nonhuman primates (Merzenich et al., 1984; Garraghty and Kaas, 1991) or removal of vibrissae in rodents (Diamond et al., 1993; Armstrong-James et al., 1994; Fox, 1994; Wallace and Fox, 1999), initiates a sequence of events in which the topographical representations of deprived and nondeprived regions of the somatosensory cortex are reorganized. These events are characterized initially by the redistribution of receptive field properties in deprived and nondeprived cortical areas (Merzenich et al., 1984; Diamond et al., 1993; Glazewski, 1998), followed by anatomical changes in the neuronal circuitry of cortical (Kossut and Juliano, 1999) and subcortical regions (Florence and Kaas, 1995; Sengelaub et al., 1997).

The mechanisms underlying experience-dependent changes in the adult cerebral cortex are at present uncertain. Nevertheless, it is generally accepted that these experience-dependent modifications are mediated by rapid changes in the synaptic efficacy of existing cortical connections, through long-term potentiation (LTP)- or long-term depression (LTD)-like processes (Donoghue, 1995). In particular, numerous studies have examined the

Received Sept. 20, 2001; revised Jan. 11, 2002; accepted Jan. 23, 2002.

This research was funded by a Natural Sciences and Engineering Research Council of Canada operating grant (R.H.D.) and graduate scholarship (C.E.B.), and a grant from the University of Calgary Research Grants Committee (R.H.D.).

Correspondence should be addressed to Dr. Richard H. Dyck, Department of Psychology, University of Calgary, 2500 University Drive, NW, Calgary, Alberta, Canada T2N IN4. E-mail: rdyck@ucalgary.ca.

Copyright (C) 2002 Society for Neuroscience $0270-6474 / 02 / 222617-09 \$ 15.00 / 0$ role of NMDA-mediated glutamatergic neurotransmission in the generation of these phenomena (Jablonska et al., 1995; Garraghty and Muja, 1996; Rema et al., 1998), because certain forms of neocortical and hippocampal LTP and LTD are dependent on these receptors (Bear and Kirkwood, 1993; Kirkwood et al., 1996; Murphy et al., 1997). The importance of glutamatergic neurotransmission in mediating experience-dependent changes in the functional organization of the cortex suggests that synaptically released zinc may also contribute to this process. Within the mammalian telencephalon, a vast network of cortically projecting glutamatergic neurons sequester zinc within their terminal boutons (Beaulieu et al., 1992; Frederickson and Moncrieff, 1994) and release it in an activity- and calcium-dependent manner (Assaf and Chung, 1984). Once released, zinc exerts potent neuromodulatory effects on both NMDA and non-NMDA receptors (Westbrook and Mayer, 1987; Christine and Choi, 1990; Smart et al., 1994; Vogt et al., 2000). Thus, because glutamatergic systems have been implicated in activity-dependent forms of cortical plasticity (i.e., LTP and LTD), it is possible that zincergic neurons may provide a mechanism that facilitates these processes.

To determine the effects of modulating sensory experience on zinc-containing axon terminals in the somatosensory cortex, we used the whisker-to-barrel pathway in adult mice. Use of this system provides some advantageous features, such as the ease with which the main sensory inputs (i.e., the vibrissae) can be manipulated and the one-to-one functional and topological correspondence between each barrel in layer IV and a particular vibrissa on the contralateral face (Woolsey and Van der Loos, 1970). Here we report that tactile experience rapidly and dynamically regulates levels of synaptic zinc in the adult somatosensory cortex. These observations suggest a potential role for synaptic 
zinc in mediating experience-dependent modifications in the synaptic organization of the adult cerebral cortex.

\section{MATERIALS AND METHODS}

Animals and treatment groups. Forty-eight male CD1 mice, between 60 and $65 \mathrm{~d}$ of age, were used to study the effects of whisker removal on levels of synaptic zinc in the adult barrel cortex. All animals were obtained from the University of Calgary Breeding Colony and maintained on standard laboratory diet and water ad libitum. Animals were group housed in clear plastic cages on a $12 \mathrm{hr}$ light/dark cycle. All experiments were conducted under the guidelines of the Canadian Council for Animal Care.

To establish the relative level of zinc staining within each barrel row in normal mice, we examined levels of zinc staining in the somatosensory cortex of unoperated control mice $(n=3)$ or from hemispheres that were taken from mice that had whiskers removed from only one side of the face $(n=4)$. From these mice, we established that levels of zinc staining in the barrel cortex of mice that had no whiskers removed was no different from that observed in hemispheres ipsilateral to the plucked side of the face. Because levels of zinc staining did not change in barrels ipsilateral to the plucked whiskers, animals in the experimental groups had whiskers removed bilaterally, and each hemisphere was considered independent in the analyses.

To investigate the potential role of synaptic zinc in experiencedependent plasticity, we examined the distribution of zinc-containing axon terminals in the somatosensory cortex of adult mice at different time points after bilateral whisker removal. Thirty mice underwent the removal (by plucking) of the first five vibrissae in row $\mathrm{C}$ (whiskers C1-C5). After this, the mice were assigned to one of seven experimental groups with survival times of $3 \mathrm{hr}(n=4), 6 \mathrm{hr}(n=4), 12 \mathrm{hr}(n=4)$, $24 \mathrm{hr}(n=6), 7 \mathrm{~d}(n=4), 14 \mathrm{~d}(n=4)$, or $21 \mathrm{~d}(n=4)$ to determine the time course of deprivation-induced changes in levels of zinc staining in row $\mathrm{C}$ of the contralateral cerebral cortex.

Additional groups of mice were used to establish whether different patterns of whisker plucking would differentially affect zinc-staining levels in barrels or rows adjacent to the deprived barrels. Here, we assessed the distribution of zinc-containing axon terminals in the barrel cortex of mice $24 \mathrm{hr}$ after having had all whiskers in rows A, B, and C removed $(n=3)$, all but the $\mathrm{C} 2$ whisker removed $(n=3)$, all row $\mathrm{D}$ whiskers removed $(n=2)$, or whiskers removed in a checkerboard or partial checkerboard pattern $(n=3)$.

To remove whiskers, mice were lightly anesthetized with halothane and gently restrained while vibrissae were plucked with surgical tweezers. Care was taken to ensure that whisker removal did not cause any excessive bleeding or damage the whisker follicle. Previous research using a similar method of whisker deprivation has shown that whisker plucking does not disrupt the integrity of the whisker follicle (Li et al., 1995). Once the mice recovered from the anesthetic, they were returned to their home cages.

Tissue preparation and staining. Histochemical localization of synaptic zinc was assessed by using the selenium method (Danscher, 1982). After the appropriate survival period, mice were administered sodium selenite $(5 \mathrm{mg} / \mathrm{ml}$ in saline; $15 \mathrm{mg} / \mathrm{kg}$, i.p.). After $60 \mathrm{~min}$, the mice were killed with an overdose of sodium pentobarbital $(100 \mathrm{mg} / \mathrm{kg})$, and the brain was removed and bisected. Cortical hemispheres were prepared for tangential sections by separating the cortex from the underlying subcortical structures and flattening them gently between two glass slides. The tissue was immediately frozen in crushed dry ice and stored at $-30^{\circ} \mathrm{C}$. Tangential sections were cut at $20 \mu \mathrm{m}$ using a cryostat and thaw mounted onto gelatin-coated glass slides. The slides were then stored at $-30^{\circ} \mathrm{C}$ in preparation for histochemical staining.

For staining, brain sections were thawed at room temperature, fixed in a descending series of ethanol (95\%, $15 \mathrm{~min} ; 70 \%, 2 \mathrm{~min} ; 50 \%, 2 \mathrm{~min})$, hydrated, and then dipped in a $0.5 \%$ gelatin solution. Selenium-bound zinc was visualized on slides by physical development in $250 \mathrm{ml}$ of developer containing $50 \%$ Gum arabic $(100 \mathrm{ml}), 2.0 \mathrm{M}$ sodium citrate buffer $(25 \mathrm{ml}), 0.5 \mathrm{M}$ hydroquinone $(30 \mathrm{ml}), 37 \mathrm{~mm}$ silver lactate $(30 \mathrm{ml})$, and distilled water $(65 \mathrm{ml})$. Sections were incubated in darkness, at room temperature, for 90-120 min. After staining, the slides were washed in running water for $20 \mathrm{~min}$ at $40^{\circ} \mathrm{C}$, then rinsed in distilled water $(2 \times 2$ $\mathrm{min}$ ) and immersed in 5\% sodium thiosulfate solution for $12 \mathrm{~min}$. Slides were then post-fixed in $70 \%$ ethanol (EtOH) for at least $30 \mathrm{~min}$, dehydrated in $95 \% \mathrm{EtOH}$ for $5 \mathrm{~min}, 100 \% \mathrm{EtOH}$ for $10 \mathrm{~min}$, cleared in xylene, and coverslipped using Permount.

Analysis of zinc-staining intensity. Levels of zinc staining in barrel cortex of control and whisker-deprived mice were determined for each hemisphere in six serial $20 \mu \mathrm{m}$ sections through layer IV of the somatosensory cortex. In each section, the entire area of the barrel field was captured digitally (COHU Model 4912 CCD; Zeiss Axioskop2 microscope; Scion LG3 Framegrabber) from which levels of zinc-staining intensity were determined densitometrically using an AppleG4 computer running Scion Image software (Scion Corporation).

In control mice, levels of zinc staining were determined densitometrically for barrel rows A through E by comparing the staining intensity of a particular row with the average staining intensity of the remaining four rows. Thus, in each section, we calculated a ratio between one row (numerator) and the remaining four rows (denominator) and referred to this ratio as the percentage difference in staining intensity for a particular row (see Fig. $1 B$ ). We used this ratio to correct for between animal differences in staining intensity.

To quantify changes in zinc staining after row $\mathrm{C}$ whisker removal, we compared the staining intensity of the deprived barrel row $\mathrm{C}$ to the average staining intensity of adjacent nondeprived barrel rows. With this method, a ratio was determined that represented the staining intensity of the deprived barrel row relative to nondeprived rows within the same brain section. The average ratio (percentage difference score) for row $\mathrm{C}$ in each hemisphere was then calculated from the section means. The mean percentage difference scores were statistically compared using a one-way ANOVA with post hoc protected $t$ tests. The significance level was set at 0.05 . All values are expressed as the mean \pm SEM.

Correlational analysis. Initial results showed that levels of zinc staining within deprived barrels decreased with longer post-plucking survival periods. This suggested to us that zinc-staining levels appeared to be correlated with neuronal activity that varied by changing whisker lengths as they regrew. To determine the relationship between whisker regrowth and levels of zinc staining, we measured the length of $\mathrm{C} 2$ whiskers as they regrew and correlated this measure with zinc-staining intensity in the $\mathrm{C} 2$ barrel hollow. The length of the whiskers at the time mice were killed was expressed as a percentage of the initial length of the whiskers. In each cortical section through layer IV of the contralateral hemisphere, we calculated the relative zinc-staining intensity in the $\mathrm{C} 2$ barrel hollow in the hemisphere contralateral to the regrown $\mathrm{C} 2$ whisker. This was done by comparing the staining intensity of the deprived $\mathrm{C} 2$ barrel hollow with the average staining intensities of adjacent nondeprived A2, B2, D2, and E2 barrel hollows. The Pearson correlation coefficient was used to assess the relationship between the length of regrown whiskers with the staining level in the corresponding $\mathrm{C} 2$ barrel hollow.

Preparation of figures. Digitally captured images were imported into Adobe Photoshop (v 5.0; Adobe Systems, San Jose, CA) where they were cropped and organized into multiplate figures. Only linear adjustments of contrast and brightness were made to the original images, and all images in a single figure were enhanced to the same degree.

\section{RESULTS}

\section{Distribution of synaptic zinc in the barrel field of control mice}

The patterned distribution of histochemically reactive zinc into barrel-like compartments was readily apparent in tangential sections through layer IV (Fig. 1A). As has been described previously in normal adult mice (Czupryn and Skangiel-Kramska, 1997; Land and Akhtar, 1999), we also found that zinc staining of the barrel field was periodically distributed, characterized by regions containing low levels of staining corresponding to barrel hollows that were separated from one another by more darkly stained inter-barrel septae. The septae appear somewhat blurred in upper layer IV but become more discrete in deeper regions.

The heterogeneous distribution of synaptic zinc within layer IV can be seen in Figure $1 A$, where five rows of barrels are oriented in a posterior to anterior manner, and lettered from A to E, with row E closest to the midline and row A the most lateral. Qualitatively, the intensity of zinc staining in barrel hollows of each row appeared homogenous, although outer rows $\mathrm{A}$ and $\mathrm{E}$ tended to stain more intensely than the inner rows B, C, and D. Our quantitative analysis of zinc staining within barrels supported this observation (Fig. 1B), indicating that the relative level of zinc 


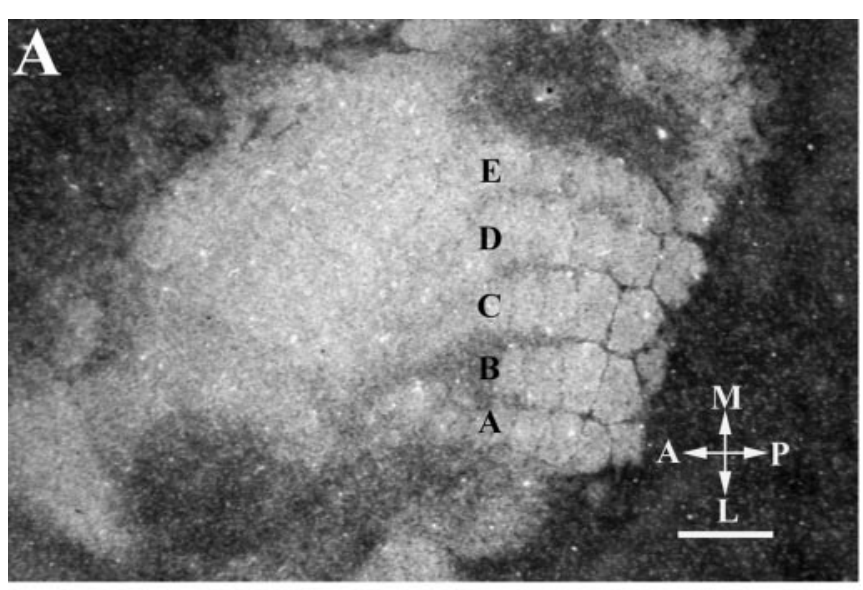

B

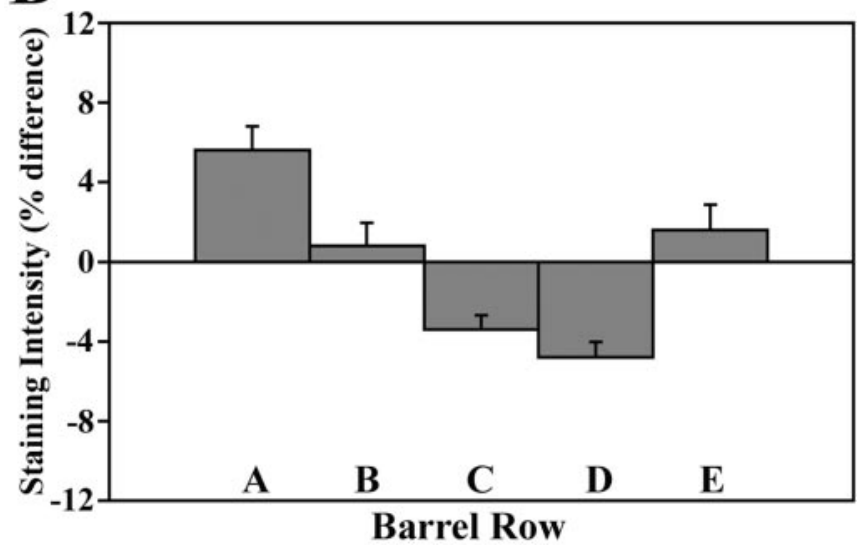

Figure 1. Distribution of histochemically reactive zinc in a section through layer IV of the somatosensory cortex in control mice $(A)$. There are five rows $(A-E)$ of whisker-related cortical barrels that delineate the location of the posteriomedial barrel subfield in the somatosensory cortex. Barrel compartments are characterized by high levels of zinc staining in inter-barrel septae and low levels of staining in barrel hollows. $A$, Anterior; $P$, posterior; $L$, lateral; $M$, medial. Scale bar, $500 \mu \mathrm{m}$. $B$, Mean $( \pm$ SEM) zinc-staining intensity for each row relative to all other rows. Note that barrel rows $A, B$, and $E$ have positive percentage difference values indicating that, on average, the staining intensity of these rows was greater than the mean staining intensity of the other rows. By contrast, negative percentage difference scores corresponding to rows $C$ and $D$ indicate that the level of zinc staining in these rows was lower than the other rows.

staining in the outer rows $\mathrm{A}$ and $\mathrm{E}$ was higher (percentage difference scores of 5.8 and $1.9 \%$, respectively) than middle rows $\mathrm{B}, \mathrm{C}$, and D. Furthermore, staining in rows $\mathrm{C}$ and $\mathrm{D}$ was lower than that of rows $\mathrm{A}, \mathrm{B}$, and $\mathrm{E}$. These low levels of staining are reflected by negative relative density values, which occur when the staining intensity of a particular row is lower than the average staining intensity of the other rows.

\section{Effect of whisker plucking}

In control mice, the relative level of zinc staining within row $\mathrm{C}$ was, on average, $3.6 \%$ lower than adjacent rows (Fig. 1B). However, examination of the relative level of zinc staining in row $\mathrm{C}$ at different time periods after removal of row $\mathrm{C}$ whiskers (Fig. 2, arrows $)$ revealed a significant effect of whisker removal $\left(F_{(7,63)}=\right.$ 20.67; $p<0.0001$ ). At $3 \mathrm{hr}$ (Fig. $2 A$ ), zinc staining increased significantly $(5.8 \% ; t=3.59 ; p<0.01)$ above control levels (Fig. 3). Six hours after whisker removal (Fig. $2 B$ ), the increase in zinc staining was $5.2 \%$ higher than control levels $(t=2.33 ; p<0.05)$
(Fig. 3). At 12 hr (Fig. 2C), 24 hr (Fig. 2D), and 7 d (Fig. 2E) after whisker removal, zinc staining within row $\mathrm{C}$ increased robustly. As shown in Figure 3, $12 \mathrm{hr}$ after whisker removal the level of zinc staining within row $\mathrm{C}$ was $16.0 \%$ above control levels $(t=$ $7.2 ; p<0.0001)$. At $24 \mathrm{hr}$ and $7 \mathrm{~d}$ after whisker removal, levels of zinc staining were $13.8 \%(t=7.37 ; p<0.001)$ and $16.9 \%(t=$ $7.65 ; p<0.001)$ higher than control levels, respectively. At survival times beyond 1 week, zinc-staining levels in deprived barrels were not significantly different from control (14 d: $t=0.77, p>$ $0.05 ; 21 \mathrm{~d}: t=0.54, p>0.05)$.

We found that changes in the levels of zinc staining resulting from whisker plucking were evident only within the deprived barrel hollows of row C. Zinc staining in the barrel septae surrounding deprived barrel hollows did not appear to be affected by whisker plucking.

\section{Are increases in zinc staining in row $\mathbf{C}$ absolute or relative?}

Recent evidence indicates that whisker removal can affect neuronal activity in cortical barrels adjacent to the deprived barrel column (Kelly et al., 1999). This finding implies that removing whiskers from row $\mathrm{C}$ may affect levels of zinc staining in adjacent nondeprived rows. As a result, this possibility calls into question the validity of our use of a relative measure to assess changes in zinc staining within row $\mathrm{C}$. To determine whether our relative measure accurately reflected increases in zinc staining after row $\mathrm{C}$ whisker removal, five mice had row $\mathrm{C}$ whiskers from one side of the face removed and were killed $24 \mathrm{hr}$ later. Thereafter, tangential sections from both hemispheres were cut and then stained for exactly the same amount of time in the same staining dish. In doing this, we were able to directly compare the staining intensity of each barrel row in the hemisphere ipsilateral to the plucked side (i.e., the control hemisphere) versus those obtained for each row in the hemisphere contralateral to the plucked side. Using a one-sample $t$ test with a Bonferroni adjustment (significance level $=0.01$ ), our results showed that the intensity of zinc staining for each of the nondeprived barrel rows (i.e., rows A, B, D, E) in the ipsilateral control hemisphere was not significantly different from the level of staining in nondeprived rows in the contralateral hemisphere. Furthermore, when examining row $\mathrm{C}$, we observed that the level of zinc staining was significantly higher in row $\mathrm{C}$ for the contralateral hemisphere $(15 \%$ increase; $t=4.75 ; p<0.01)$ than in row $\mathrm{C}$ for the ipsilateral control hemisphere, exactly the same values that are obtained with bilateral plucking. These results not only validate our use of a ratio (i.e., percentage difference score) to quantify relative changes in zinc staining, but also show that higher levels of staining in row $\mathrm{C}$ reflect an absolute increase in staining intensity.

\section{Other patterns of whisker plucking}

Previous work has shown that the degree and form of whisker deprivation plasticity is highly dependent on the spatial relationship of whiskers that are removed (Wallace and Fox, 1999). To determine whether the spatial extent of deprivation-induced changes in zinc staining would be affected by different patterns of plucking, we removed all whiskers from row $\mathrm{D}$, or rows $\mathrm{A}, \mathrm{B}$, and $\mathrm{C}$, or all whiskers but $\mathrm{C} 2$, or the whiskers were removed in a checkerboard pattern. All animals were then allowed to survive for $24 \mathrm{hr}$. Removal of whiskers from rows A, B, and C resulted in a robust increase in the density of staining for histochemically reactive zinc in barrel hollows associated only with the plucked whiskers (Fig. 4A). Similarly, removal of all whiskers except the 

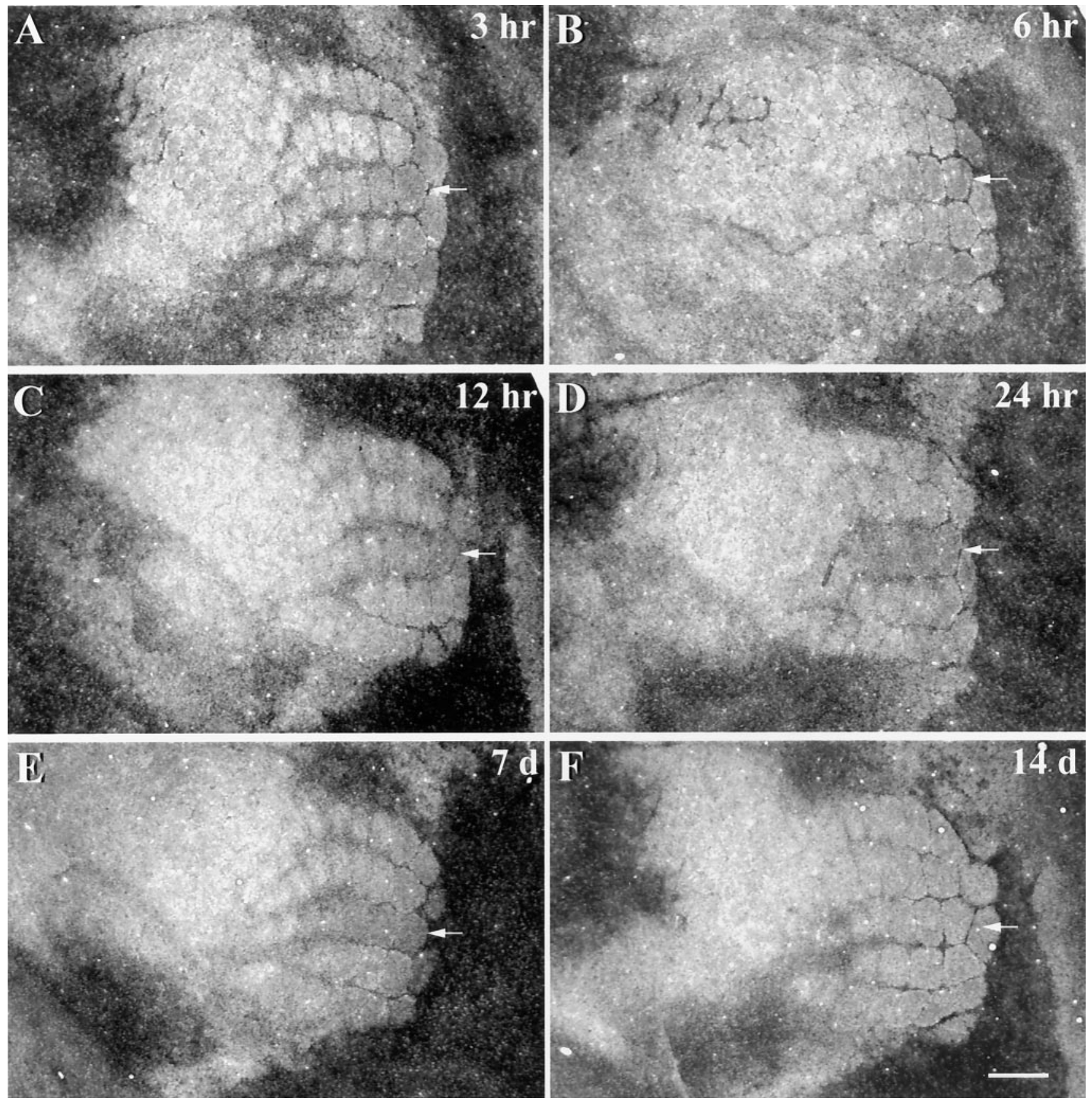

Figure 2. Changes in zinc staining in row $\mathrm{C}$ at survival times ranging from $3 \mathrm{hr}$ to 2 weeks after the removal of whiskers from row $\mathrm{C}$ of the contralateral face. The position of row $\mathrm{C}$ is indicated by the white arrow in each panel. Subtle increases in zinc staining were apparent in row $\mathrm{C}$ within $3 \mathrm{hr}$ of whisker removal $(A)$. At $6(B), 12(C)$, and $24 \mathrm{hr}(D)$, and 1 week $(E)$ after whisker removal, levels of zinc staining in the deprived row $\mathrm{C}$ were robustly increased relative to adjacent nondeprived barrel rows. Two weeks after whiskers were removed $(F)$, levels of zinc staining in row $\mathrm{C}$ were normal. Scale bar, $500 \mu \mathrm{m}$.

C2 whisker markedly increased the density of zinc staining within deprived barrel hollows, relative to that corresponding to the intact C2 whisker (Fig. 4B). In fact, regardless of the deprivation pattern, increases in zinc staining were confined only to the deprived barrel hollows, those that corresponded to the plucked whisker(s) on the contralateral face (other patterns not shown).

To further characterize activity-dependent changes in zinc levels, we examined zinc staining in nondeprived and deprived barrel hollows at higher magnification (Figs. $4 C, D$ ). Because zinc staining is restricted to the axon terminals of zinc-ergic neurons (Beaulieu et al., 1992; Frederickson and Moncrieff, 1994), high- magnification photomicrographs revealed that the histochemical reaction product consisted of numerous black punctae that are found either in small singular spots or in larger irregular clusters. In the nondeprived barrel hollows (Fig. $4 C$ ), the majority of zinc stained punctae occurred in singular spots, interspersed with a few more larger, irregularly shaped clusters. In contrast, zincstained punctae in the deprived D2 barrel hollow (Fig. 4D) appeared much more numerous and more densely clustered than in the nondeprived barrel hollow (Fig. $4 C$ ). These results suggest that higher levels of zinc staining in deprived barrel hollows reflect an increase in the density of zinc-stained punctae. 


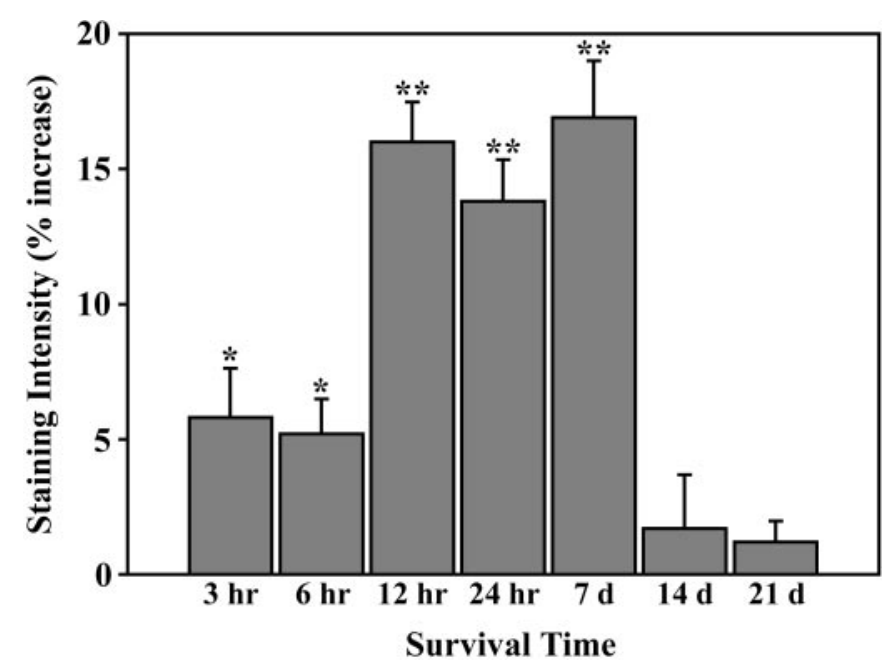

Figure 3. Quantitative analysis of zinc staining in barrel row $\mathrm{C}$ at different survival times after removal of row $\mathrm{C}$ whiskers. Relative to control mice, levels of zinc staining increased significantly at $3,6,12$, and $24 \mathrm{hr}$, and 1 week after whisker plucking. However, with longer survival times ( 2 and 3 weeks), the level of zinc staining in row $\mathrm{C}$ was not significantly different from baseline levels. ${ }^{*} p<0.05 ; * * p<0.001$.

Whether this results from an increase in the number of zinccontaining vesicles per terminal or an increase in the level of zinc in individual vesicles remains to be established.

\section{Relationship to whisker length}

The observation that longer survival periods were associated with normalized levels of zinc staining in deprived barrel hollows prompted us to associate the levels of zinc staining in deprived barrel hollows with the length of whiskers as they regrew. The results of our analysis indicated a highly significant linear relationship between levels of zinc staining in deprived barrel hollows and the length of regrown whiskers (Fig. 5) $(r=-0.81 ; p<$ 0.001). Specifically, whisker length was inversely related to the level of zinc staining within the deprived barrel hollow. Thus, greater increases in zinc staining were associated with whiskers that had undergone less regrowth, whereas more fully regrown whiskers were associated with much smaller increases in staining. Furthermore, increased levels of zinc staining appeared to be directly related to the regrowth of the whisker, rather than to the length of survival time after whisker removal. Supporting this assertion was the observation that some mice from the 1 week group exhibited greater whisker regrowth and had smaller increases in zinc staining than animals from the 3 week group that had less regrowth but higher levels of zinc staining. Our results show that levels of zinc staining in barrel hollows are directly proportional to the length of regrowing whiskers.

\section{DISCUSSION}

Zinc-selenide histochemistry was used to visualize zinccontaining axon terminals in layer IV of the adult mouse somatosensory cortex and to determine the extent to which levels of synaptic zinc are regulated by sensory input. In the barrel field of normal mice, zinc staining demarcates barrel compartments with high levels of staining in the inter-barrel septae and low levels of staining in barrel hollows. However, when vibrissae were removed, the intensity of zinc staining within deprived barrel hollows increased significantly. This increase was evident within $3 \mathrm{hr}$ of whisker removal and persisted for 1 week. With longer survival times, previously plucked whiskers began to regrow, and this regrowth was accompanied by a gradual decline in levels of zinc staining, reaching normal levels by 2 weeks after whisker removal. The level of zinc staining in deprived barrel hollows was inversely and linearly related to the length of regrowing whiskers.

\section{Source of zinc-ergic innervation}

An important question that arises out of the present results relates to the locus of change in zinc levels. Histochemical studies characterizing the distribution of synaptic zinc in the somatosensory cortex of rodents have shown that zinc-containing terminals are distributed heterogeneously across cortical laminas, with highest densities in supragranular and infragranular layers (Czupryn and Skangiel-Kramska, 1997; Land and Akhtar, 1999). However, despite the significantly greater zinc-ergic innervation of supragranular and infragranular layers, we observed experiencedependent changes in zinc staining only within the thalamic recipient barrel hollows of layer IV. The fact that layer IV is a major termination site for glutamatergic neurons from the thalamus (Koralek et al., 1988, 1990; Kharazia and Weinberg, 1994), and that zinc-ergic neurons comprise a subset of glutaminergic neurons, raises the possibility that increased levels of zinc staining seen in zinc-ergic terminals in layer IV might originate from neurons in the thalamus. Although not addressed in the present study, there is substantial evidence to suggest the contrary, i.e., that changes in zinc staining originate from corticocortical rather than thalamocortical projection neurons. First, studies using retrograde tracing techniques to visualize zinc-containing cell bodies and their axonal projections have failed to reveal labeled cell bodies in nuclei of the thalamus projecting to somatosensory cortex. Instead, zinc-ergic neurons were found predominately in cortical laminas II, III, and VI and appeared to send and receive ipsilateral and transcallosal corticocortical projections (Garrett and Slomianka, 1992; Garrett et al., 1992; Dyck and O'Leary, 1995; Casanovas-Aguilar et al., 1998). Second, recent work examining the distribution of neuronal somata expressing mRNA for $\mathrm{ZnT}-3$, a putative transporter involved in the uptake of zinc into synaptic vesicles, has found that cells expressing this mRNA are abundant in the hippocampus and cortex but appear to be mostly absent in the thalamus (Palmiter et al., 1996). Taken together, these observations suggest that the population of zinc-ergic neurons that are modulated by sensory experience are of cortical and not thalamic origin.

\section{Comparison with previous literature}

Our observation that histochemical staining for synaptic zinc in the somatosensory cortex is dramatically altered by changes in sensory experience is in accordance with a report by Dyck et al. (1994) showing that monocular deprivation rapidly regulates levels of synaptic zinc in the adult primate visual cortex. However, the findings of the present study contrast with previous investigations in the rodent barrel cortex, showing that whisker removal has no effect on histochemical staining for synaptic zinc in the adult cortex (Land and Akhtar, 1999; Quaye et al., 1999, Czupryn and Skangiel-Kramska, 2001). To address this discrepancy, we point out that there were two important differences in the methodologies used in previous studies compared with the present one. First, in previous studies, whiskers were trimmed chronically for longer periods (up to 3-6 weeks), starting from 6 to 10 weeks of age in mice (Quaye et al., 1999; Czupryn and SkangielKramska, 2001) and 2 months of age in rats (Land and Akhtar, 1999). Because the stain used in those studies and the present one 

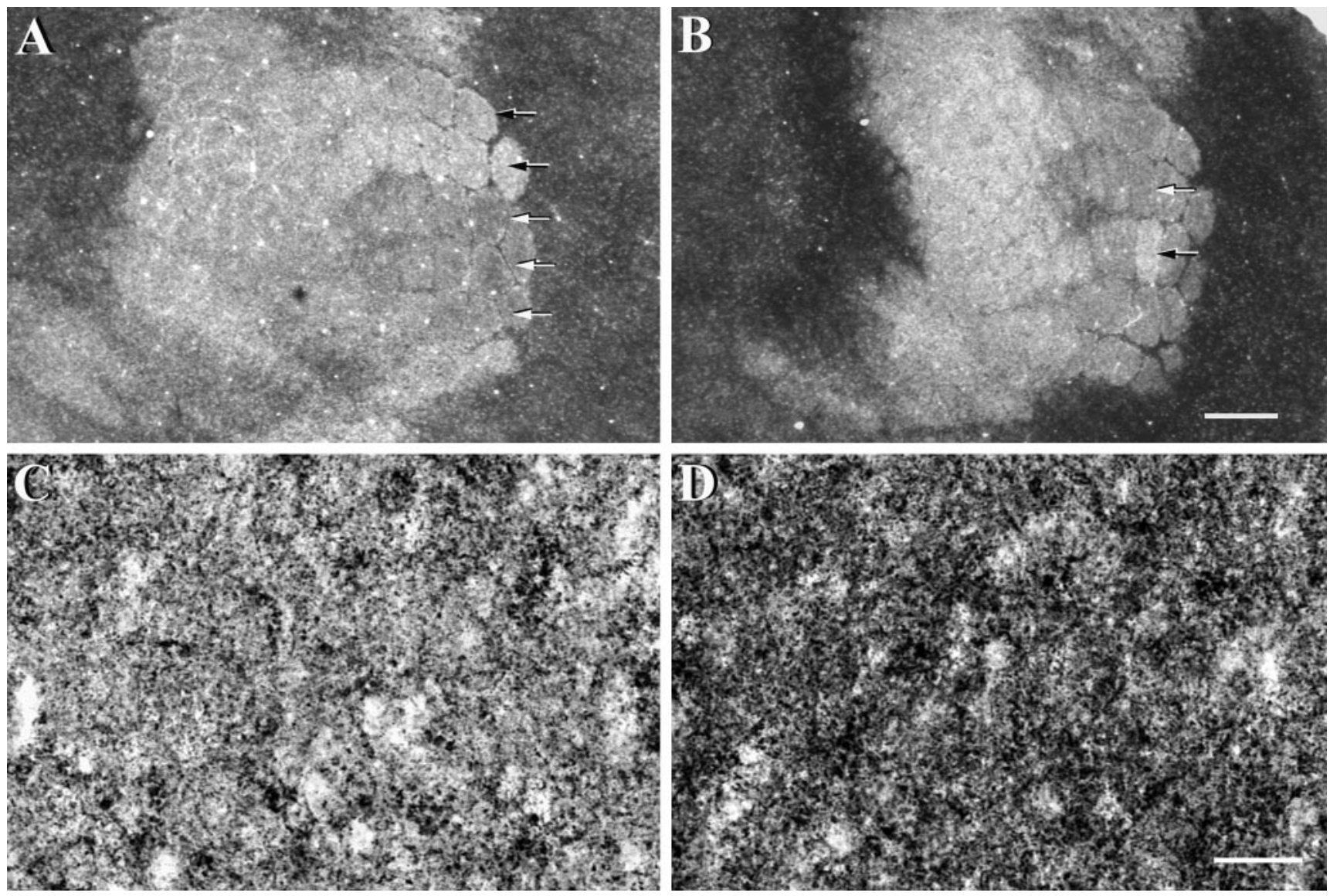

Figure 4. Alterations to zinc staining levels in somatosensory cortex $24 \mathrm{hr}$ after other patterns of whisker removal. Animals either had rows A, B, and $\mathrm{C}$ removed $(A)$ or had all but the $\mathrm{C} 2$ whisker removed $(B)$. Zinc staining in cortical barrels associated with plucked whiskers (white arrows) appeared much darker than that observed for nondeprived barrels (black arrows). At higher magnification, zinc staining appeared punctate in both nondeprived $(C)$ and deprived $(D)$ barrel hollows. In deprived barrel hollows $(D)$, zinc-stained punctae appeared much more numerous and more densely clustered than that observed in the nondeprived barrel hollow $(C)$. Scale bar (shown in $B$ ): $A, B, 500 \mu \mathrm{m}$; (shown in $D$ ): $C, D, 20 \mu \mathrm{m}$.

is selective for zinc in axon terminals, it is possible that after several weeks of sensory deprivation, the uptake and release of zinc within axon terminals may have returned to normal levels. Second, the animals in previous studies were allowed to survive for up to 1 month after chronic whisker trimming, allowing whiskers to regrow to normal lengths. As a result, histochemical changes in synaptic zinc would have occurred shortly after whisker trimming but returned to normal levels by the time of kill. This seems most likely considering our present results which demonstrate that staining for synaptic zinc in deprived barrel hollows appears normal once whiskers have regrown.

Electrophysiological studies have demonstrated that the functional organization of the adult cerebral cortex is rapidly modified by changes in sensory experience (Gilbert and Wiesel, 1992; Buonomano and Merzenich, 1998). For example, the receptive field properties of neurons in the primary somatosensory cortex of rodents can be modulated after only a few hours of altered sensory experience (Diamond et al., 1994; Rema and Ebner, 1999; Barth et al., 2000). In an effort to understand the underlying molecular correlates of these physiological changes, several authors have examined the effects of altered sensory experience on the expression levels of a number of neuroactive molecules. In the rodent somatosensory cortex, long periods of whisker deprivation have been shown to reduce the expression of cytochrome oxidase (Land and Simons, 1985), glutamic acid decarboxylase (GAD)
(Welker et al., 1989; Akhtar and Land, 1991), GABA (Micheva and Beaulieu, 1995), and GABA-A receptors (Skangiel-Kramska et al., 1994; Land et al., 1995; Fuchs and Salazar, 1998). Comparably, in the primary visual cortex of cats and monkeys, monocular deprivation decreases the expression of cytochrome oxidase (Hevner and Wong-Riley, 1990), glutamate (Carder and Hendry, 1994), GAD (Hendry and Jones, 1988), GABA (Hendry and

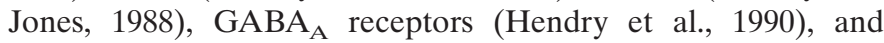
NMDA receptor subunits (Catalano et al., 1997) while increasing the expression of calmodulin-dependent protein kinase (Hendry and Kennedy, 1986) and neurotrophic factors (Obata et al., 1999) in cortical domains corresponding to the deprived input. However, in each of these studies, deprivation periods of several days to several weeks were required to affect the expression of these molecules.

Despite the search, histochemical markers with expression patterns that correlate temporally and spatially with rapid (i.e., within minutes to hours) electrophysiological changes in the cortex have remained elusive. At present, only NMDA receptor subunits (Quinlan et al., 1999), immediate early genes (Rosen et al., 1992; Beaver et al., 1993), and transcription factors such as Zif268 (Chaudhuri and Cynader, 1993) and cAMP response element binding protein (Barth et al., 2000) have been shown to modify their expression levels after only a few hours of altered sensory experience. Perhaps the most intriguing aspect of the 


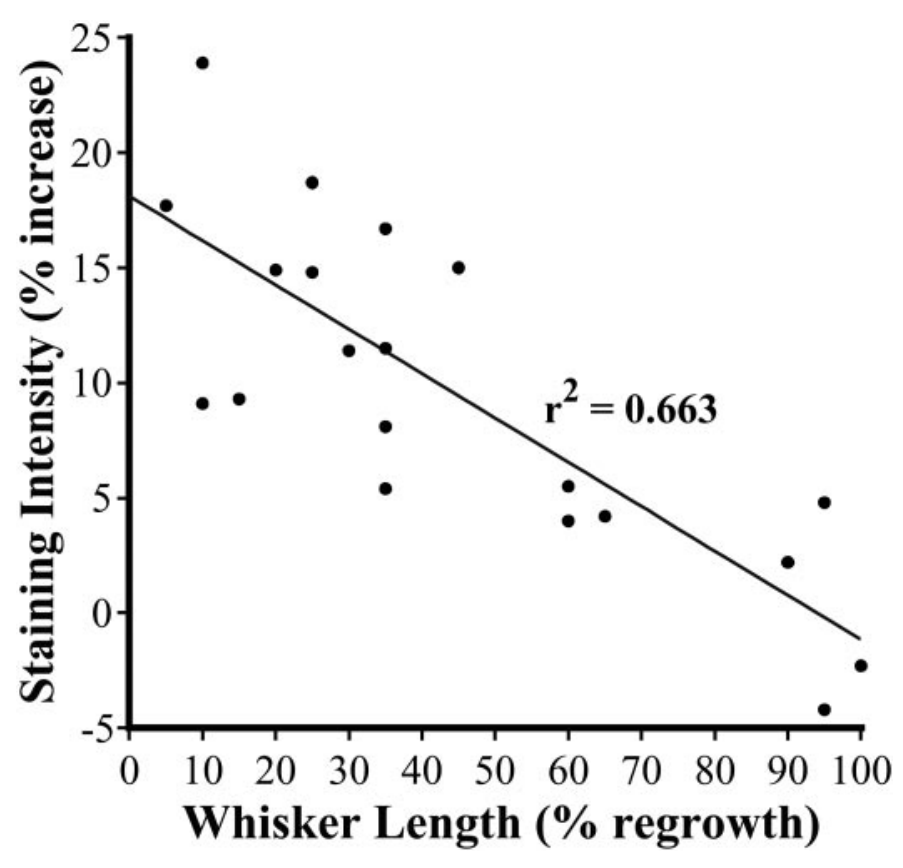

Figure 5. Scatterplot showing the inverse relationship between levels of zinc staining in the deprived barrel hollow and the length of the regrowing whisker. This significant correlation $\left(r^{2}=0.66 ; p<0.001\right)$ demonstrates that higher levels of zinc staining in deprived barrel hollows are associated with shorter whiskers, whereas fully regrown whiskers are associated with a return of zinc staining to normal levels.

present study was the rapidity with which cortical levels of synaptic zinc could be regulated by sensory experience. We have demonstrated that just $3 \mathrm{hr}$ of sensory deprivation was sufficient to significantly alter levels of zinc staining in deprived regions of cortex. Although indefinite from our results, it is tempting to speculate that levels of synaptic zinc may be altered within minutes after changing sensory experience. However, to verify this claim, future experiments involving real-time measurements of synaptic zinc in the cortex will be necessary. Nevertheless, our results provide a novel anatomical correlate of rapid, experiencedependent synaptic changes in the adult cortex.

\section{Functional implications}

Two important questions arise from our findings. The first relates to the mechanism responsible for modulating levels of zinc within individual axon terminals in an experience- or activity-dependent manner. The second, arguably more important question pertains to whether these increases have functional consequences for synaptic transmission in the cerebral cortex. To address the first question, we could argue that increased levels of zinc staining in deprived barrel columns reflect activity-dependent changes in the release and uptake of zinc into axon terminals. Previous work that has assessed the kinetics of zinc turnover suggests that zinc is released in an activity-dependent manner (Assaf and Chung, 1984). Once released, extracellular concentrations of zinc are regulated by transporters that facilitate the re-uptake of zinc into the presynaptic terminal (Palmiter et al., 1996; Cole et al., 1999). However, if the release of zinc is activity dependent but the uptake is not, then it would seem plausible that in situations that result in decreased afferent neuronal activity in the cortex, such as that caused by whisker removal (Durham and Woolsey, 1978; Kelly et al., 1999), one disrupts zinc homeostasis such that more zinc is taken up into zinc-ergic axon terminals than is released.
Future experiments exploring this interaction between neuronal activity and the efficacy of zinc transporters will be necessary to resolve this question.

Alternatively, modulations of the presynaptic zinc levels might contribute, mechanistically, to experience-dependent changes of the synaptic organization of the cerebral cortex. Current hypotheses for mechanisms supporting experience-dependent plasticity in the cortex suggest that NMDA receptor-dependent forms of LTP and LTD might play an important role in this phenomenon (Artola and Singer, 1987; Castro-Alamancos et al., 1995; Kirkwood et al., 1996; Feldman, 2000). The appeal of NMDAdependent LTP and LTD as processes that mediate experiencedependent plasticity is attributable in part to the fact that (1) NMDA-dependent LTP and LTD can be readily induced in primary sensory regions of the cortex (Bear and Kirkwood, 1993; Donoghue, 1995), (2) manipulations of sensory experience can produce LTP- and LTD-like changes in the response properties of cortical neurons (Diamond et al., 1993, 1994; Wallace and Fox, 1999), and (3) pharmacological blockade of NMDA receptors disrupts experience-dependent reorganizations of the synaptic organization of the cortex (Jablonska et al., 1995; Garraghty and Muja, 1996; Rema et al., 1998). With this in mind, it is possible that activity-dependent changes in presynaptic levels of zinc may provide a substrate for these processes to occur (Weiss et al., 1989). Although the precise physiological role of synaptic zinc is unknown, studies have demonstrated that zinc is capable of modulating NMDA-dependent forms of LTP and LTD in the CA1 and CA3 regions of the hippocampus (Xie and Smart, 1994; Lu et al., 2000; Li et al., 2001). Synaptic zinc seems well positioned to function as a mediator of rapid changes in synaptic efficacy by virtue of its potent neuromodulatory effects on NMDA and non-NMDA receptor-mediated glutamatergic neurotransmission (Westbrook and Mayer, 1987; Christine and Choi, 1990; Smart et al., 1994; Vogt et al., 2000). In addition, zinc can regulate the activation of a number of other ligand-gated receptors, including the $\alpha-7$ nicotinic (Palma et al., 1998), 5-HT(3) (Hubbard and Lummis, 2000), $\mathrm{GABA}_{\mathrm{A}}$, and $\mathrm{GABA}_{\mathrm{B}}$ receptor subtypes (Smart et al., 1994).

A central theme of models concerned with experiencedependent changes in the cortex is that postsynaptic levels of calcium must be modified to induce a cascade of molecular changes that are required for the induction of LTP and LTD (Artola and Singer, 1993; Benuskova et al., 2001). The fact that zinc can regulate NMDA and AMPA receptor-gated channels suggests that experience-dependent changes in synaptic zinc may modulate postsynaptic calcium influx and in turn affect the activity of calcium-dependent proteins. Alternatively, zinc is known to compete with calcium for postsynaptic entry routes through NMDA receptors, calcium-permeable AMPA receptors, and voltage-gated calcium channels (Choi and Koh, 1998). After postsynaptic entry, zinc itself is capable of modulating the activity of protein kinases such as PKC (Baba et al., 1991), Src (Zheng et al., 1998), and calcium/calmodulin-dependent kinase II (Weinberger and Rostas, 1991; Lengyel et al., 2000). The activation of these proteins has been shown to play an important role in the induction of LTP (Malenka et al., 1989; Lu et al., 1998) and may participate in barrel cortex plasticity (Glazewski et al., 2000). Very recently, in fact, long-term potentiation at mossy fiber-CA3 synapses has been found to require zinc translocation ( $\mathrm{Li}$ et al., 2001). Further experiments are necessary to establish whether synaptic zinc is an active contributor to activity- and experiencedependent forms of synaptic plasticity in the barrel cortex. 


\section{REFERENCES}

Akhtar ND, Land PW (1991) Activity-dependent regulation of glutamic acid decarboxylase in the rat barrel cortex: effects of neonatal versus adult sensory deprivation. J Comp Neurol 307:200-213.

Armstrong-James M, Diamond ME, Ebner FF (1994) An innocuous bias in whisker use in adult rats modifies receptive fields of barrel cortex neurons. J Neurosci 14:6978-6991.

Artola A, Singer W (1987) Long-term potentiation and NMDA receptors in rat visual cortex. Nature 330:649-652.

Artola A, Singer W (1993) Long-term depression of excitatory synaptic transmission and its relationship to long-term potentiation. Trends Neurosci 16:480-487.

Assaf SY, Chung SH (1984) Release of endogenous Zn2+ from brain tissue during activity. Nature 308:734-736.

Baba A, Etoh S, Iwata H (1991) Inhibition of NMDA-induced protein kinase $\mathrm{C}$ translocation by a $\mathrm{Zn}^{2+}$ chelator: implication of intracellular $\mathrm{Zn}^{2+}$. Brain Res 557:103-108.

Barth AL, McKenna M, Glazewski S, Hill P, Impey S, Storm D, Fox K (2000) Upregulation of cAMP response element-mediated gene expression during experience-dependent plasticity in adult neocortex. J Neurosci 20:4206-4216.

Bear MF, Kirkwood A (1993) Neocortical long-term potentiation. Curr Opin Neurobiol 3:197-202.

Beaulieu C, Dyck R, Cynader M (1992) Enrichment of glutamate in zinc-containing terminals of the cat visual cortex. NeuroReport 3:861-864.

Beaver CJ, Mitchell DE, Robertson HA (1993) Immunohistochemical study of the pattern of rapid expression of c-fos protein in the visual cortex of dark-reared kittens following initial exposure to light. J Comp Neurol 333:469-484.

Benuskova L, Rema V, Armstrong-James M, Ebner FF (2001) Theory for normal and experience-dependent plasticity in neocortex of adult rats. Proc Natl Acad Sci USA 98:2797-2802.

Buonomano DV, Merzenich MM (1998) Cortical plasticity: from synapses to maps. Annu Rev Neurosci 21:149-186.

Carder RK, Hendry SH (1994) Neuronal characterization, compartmental distribution, and activity- dependent regulation of glutamate immunoreactivity in adult monkey striate cortex. J Neurosci $14: 242-262$

Casanovas-Aguilar C, Reblet C, Perez-Clausell J, Bueno-Lopez JL (1998) Zinc-rich afferents to the rat neocortex: projections to the visual cortex traced with intracerebral selenite injections. J Chem Neuroanat 15:97-109.

Castro-Alamancos MA, Donoghue JP, Connors BW (1995) Different forms of synaptic plasticity in somatosensory and motor areas of the neocortex. J Neurosci 15:5324-5333.

Catalano SM, Chang CK, Shatz CJ (1997) Activity-dependent regulation of NMDAR1 immunoreactivity in the developing visual cortex. J Neurosci 17:8376-8390.

Chaudhuri A, Cynader MS (1993) Activity-dependent expression of the transcription factor Zif268 reveals ocular dominance columns in monkey visual cortex. Brain Res 605:349-353.

Choi DW, Koh JY (1998) Zinc and brain injury. Annu Rev Neurosci 21:347-375.

Christine CW, Choi DW (1990) Effect of zinc on NMDA receptormediated channel currents in cortical neurons. J Neurosci 10:108-116.

Cole TB, Wenzel HJ, Kafer KE, Schwartzkroin PA, Palmiter RD (1999) Elimination of zinc from synaptic vesicles in the intact mouse brain by disruption of the ZnT3 gene. Proc Natl Acad Sci USA 96:1716-1721.

Czupryn A, Skangiel-Kramska J (1997) Distribution of synaptic zinc in the developing mouse somatosensory barrel cortex. J Comp Neurol 386:652-660.

Czupryn A, Skangiel-Kramska J (2001) Deprivation and denervation differentially affect zinc-containing circuitries in the barrel cortex of mice. Brain Res Bull 15:287-295.

Danscher G (1982) Exogenous selenium in the brain. A histochemical technique for light and electron microscopical localization of catalytic selenium bonds. Histochemistry 76:281-293.

Diamond ME, Armstrong-James M, Ebner FF (1993) Experiencedependent plasticity in adult rat barrel cortex. Proc Natl Acad Sci USA 90:2082-2086.

Diamond ME, Huang W, Ebner FF (1994) Laminar comparison of somatosensory cortical plasticity. Science 265:1885-1888.

Donoghue JP (1995) Plasticity of adult sensorimotor representations. Curr Opin Neurobiol 5:749-754.

Durham D, Woolsey TA (1978) Acute whisker removal reduces neuronal activity in barrels of mouse SmI cortex. J Comp Neurol 178:629-644

Dyck RH, O'Leary DDM (1995) Origin and ontogeny of zinc-ergic inputs to rat somatosensory cortex. Soc Neurosci Abstr 21:571.

Dyck RH, Chaudhuri A, Cynader MS (1994) Activity-dependent columnar regulation of zinc in the adult primate visual cortex. Invest Ophthal Vis Sci 35:1971.
Feldman DE (2000) Timing-based LTP and LTD at vertical inputs to layer II/III pyramidal cells in rat barrel cortex. Neuron 27:45-56.

Florence SL, Kaas JH (1995) Large-scale reorganization at multiple pathways of the somatosensory pathway follows therapeutic amputation of the hand in monkeys. J Neurosci 15:8083-8095.

Fox K (1994) The cortical component of experience-dependent synaptic plasticity in the rat barrel cortex. J Neurosci 14:7665-7679.

Frederickson CJ, Moncrieff DW (1994) Zinc-containing neurons. Biol Signals 3:127-139.

Fuchs JL, Salazar E (1998) Effects of whisker trimming on GABA-A receptor binding in the barrel cortex of developing and adult rats. J Comp Neurol 395:209-216.

Garraghty PE, Kaas JH (1991) Large-scale functional reorganization in adult monkey cortex after peripheral nerve injury. Proc Natl Acad Sci USA 88:6976-6980.

Garraghty PE, Muja N (1996) NMDA receptors and plasticity in adult primate somatosensory cortex. J Comp Neurol 367:319-326.

Garrett B, Slomianka L (1992) Postnatal development of zinc-containing cells and neuropil in the visual cortex of the mouse. Anat Embryol 186:487-496.

Garrett B, Sorensen JC, Slomianka L (1992) Fluoro-Gold tracing of zinc-containing afferent connections in the mouse visual cortices. Anat Embryol 185:451-459.

Gilbert CD, Wiesel TN (1992) Receptive field dynamics in adult primary visual cortex. Nature 356:150-152.

Glazewski S (1998) Experience-dependent changes in vibrissae evoked responses in the rodent barrel cortex. Acta Neurobiol Exp 58:309-320.

Glazewski S, Giese KP, Silva, Fox K (2000) The role of $\alpha$-CaMKII autophosphorylation in neocortical experience-dependent plasticity. Nat Neurosci 3:911-918.

Hendry SHC, Jones EG (1988) Activity-dependent regulation of GABA expression in the visual cortex of adult monkeys. Neuron 1:701-712.

Hendry SHC, Kennedy MB (1986) Immunoreactivity for a calmodulindependent protein kinase is selectively increased in macaque striate cortex after monocular deprivation. Proc Natl Acad Sci USA $83: 1536-1540$.

Hendry SHC, Fuchs J, De Blas AL, Jones EG (1990) Distribution and plasticity of immunocytochemically localized $\mathrm{GABA}_{\mathrm{A}}$ receptors in adult monkey visual cortex. J Neurosci 10:2438-2450.

Hevner RF, Wong-Riley MTT (1990) Regulation of cytochrome oxidase protein levels by functional activity in the macaque monkey visual system. J Neurosci 10:1331-1340.

Hubbard PC, Lummis SC (2000) Zn(2+) enhancement of the recombinant 5 -HT(3) receptor is modulated by divalent cations. Eur J Pharmacol 394:189-197.

Jablonska B, Gierdalski M, Siucinska E, Skangiel-Kramska J, Kossut M (1995) Partial blocking of NMDA receptors restricts plastic changes in adult mouse barrel cortex. Behav Brain Res 66:207-216.

Kelly MK, Carvell GE, Kodger JM, Simons DJ (1999) Sensory loss by selected whisker removal produces immediate disinhibition in the somatosensory cortex of behaving rats. J Neurosci 19:9117-9125.

Kirkwood A, Rioult MC, Bear MF (1996) Experience-dependent modification of synaptic plasticity in visual cortex. Nature 381:526-528.

Kharazia VN, Weinberg RJ (1994) Glutamate in thalamic fibers terminating in layer IV of primary sensory cortex. J Neurosci 14:6021-6032.

Koralek KA, Jensen KF, Killackey HP (1988) Evidence for two complementary patterns of thalamic input to the rat somatosensory cortex. Brain Res 463:346-351.

Koralek KA, Olavarria J, Killackey HP (1990) Areal and laminar organization of corticocortical projections in the rat somatosensory cortex. J Comp Neurol 299:133-150.

Kossut M, Juliano SL (1999) Anatomical correlates of representational map reorganization induced by partial vibrissectomy in the barrel cortex of adult mice. Neuroscience 92:807-817.

Land PW, Akhtar ND (1999) Experience-dependent alteration of synaptic zinc in rat somatosensory barrel cortex. Somatosens Mot Res 16:139-150.

Land PW, Simons DJ (1985) Metabolic activity in SmI cortical barrels of the adult rats is dependent on patterned sensory stimulation of the mystacial vibrissae. Brain Res 341:189-194.

Land PW, De Blas AL, Reddy N (1995) Immunocytochemical localization of GABA receptors in rat somatosensory cortex and effects of tactile deprivation. Somatosens Motor Res 12:127-141.

Lengyel I, Fieuw-Makaroff S, Hall AL, Sim ATR, Rostas JAP, Dunkley PR (2000) Modulation of the phosphorylation and activity of calcium/ calmodulin-dependent protein kinase II by zinc. J Neurochem 75:594-605

Li X, Glazewski S, Lin X, Elde R, Fox K (1995) Effect of vibrissae deprivation on follicle innervation, neuropeptide synthesis in the trigeminal ganglion, and S1 barrel cortex plasticity. J Comp Neurol $357: 465-481$

Li Y, Hough CJ, Frederickson CJ, Sarvey JM (2001) Induction of mossy fiber-CA3 long-term potentiation requires translocation of synaptically released $\mathrm{Zn}^{2+}$. J Neurosci 21:8015-8025.

Lu YM, Roder JC, Davidow J, Salter MW (1998) Src activation in the 
induction of long-term potentiation in CA1 hippocampal neurons. Science 279:1363-1368.

Lu YM, Taverna FA, Tu R, Ackerly CA, Wang YT, Roder J (2000) Endogenous $\mathrm{Zn}(2+)$ is required for the induction of long-term potentiation in rat hippocampal mossy fiber-CA3 synapses. Synapse 38:187-197.

Malenka RC, Kauer JA, Perkel DJ, Mauk MD, Kelly PT, Nicoll RA, Waxham MN (1989) An essential role for postsynaptic calmodulin and protein kinase activity in long-term potentiation. Nature 340:554-557.

Merzenich MM, Nelson RJ, Stryker MP, Cynader MS, Schoppmann A, Zook JM (1984) Somatosensory cortical map changes following digit amputation in adult monkeys. J Comp Neurol 224:591-605.

Micheva KD, Beaulieu C (1995) Neonatal sensory deprivation induces selective changes in the quantitative distribution of GABAimmunoreactive neurons in the rat barrel field cortex. J Comp Neurol 361:574-584.

Murphy KP, Reid GP, Trentham DR, Bliss TV (1997) Activation of NMDA receptors is necessary for the induction of associative longterm potentiation in area CA1 of the rat hippocampal slice. J Physiol (Lond) 504:379-385.

Obata S, Obata J, Das A, Gilbert CD (1999) Molecular correlates of topographic reorganization in primary visual cortex following retinal lesions. Cereb Cortex 9:238-248.

Palma E, Maggi L, Miledi R, Eusebi F (1998) Effects of Zn2+ on wild and mutant neuronal alpha-7 nicotinic receptors. Proc Natl Acad Sci USA 95:10246-10250.

Palmiter RD, Cole TB, Quaife CJ, Findley SD (1996) ZnT-3, a putative transporter of zinc into synaptic vesicles. Proc Natl Acad Sci USA 93:14934-14939.

Quaye VL, Shamalla-Hannah L, Land PW (1999) Experiencedependent alteration of zinc-containing circuits in somatosensory cortex of the mouse. Brain Res Dev Brain Res 114:283-287.

Quinlan EM, Philpot BD, Huganir RL, Bear MF (1999) Rapid, experience-dependent expression of synaptic NMDA receptors in visual cortex in vivo. Nat Neurosci 2:352-357.

Rema V, Ebner FF (1999) Effect of enriched environment rearing on impairments in cortical excitability and plasticity after prenatal alcohol exposure. J Neurosci 19:10993-11006.

Rema V, Armstrong-James M, Ebner FF (1998) Experience-dependent plasticity of adult rat S1 cortex requires local NMDA receptor activation. J Neurosci 18:10196-10206.
Rosen KM, McCormack MA, Villa-Komaroff L, Mower GD (1992) Brief visual experience induces immediate early gene expression in the cat visual cortex. Proc Natl Acad Sci USA 89:5437-5441.

Sengelaub DR, Muja N, Mills AC, Myers WA, Churchill JD, Garraghty PE (1997) Denervation-induced sprouting of intact peripheral afferents into the cuneate nucleus of adult rats. Brain Res 769:256-262.

Skangiel-Kramska J, Glazewski S, Jablonska B, Siucinska E, Kossut M (1994) Reduction of $\mathrm{GABA}_{\mathrm{A}}$ receptor binding of $\left[{ }^{3} \mathrm{H}\right]$ muscimol in the barrel field of mice after peripheral denervation: transient and longlasting effects. Exp Brain Res 100:39-46.

Smart TG, Xie X, Krishek BJ (1994) Modulation of inhibitory and excitatory amino acid receptor ion channels by zinc. Prog Neurobiol 42:393-341.

Trachtenberg JT, Trepel C, Stryker MP (2000) Rapid extragranular plasticity in the absence of thalamocortical plasticity in the developing primary visual cortex. Science 287:2029-2032.

Vogt K, Mellor J, Tong G, Nicoll R (2000) The actions of synaptically released zinc at hippocampal mossy fiber synapses. Neuron 26:187-196.

Wallace H, Fox K (1999) Local cortical interactions determine the form of cortical plasticity. J Neurobiol 41:58-63.

Weinberger RP, Rostas JA (1991) Effect of zinc on calmodulinstimulated protein kinase II and protein phosphorylation in rat cerebral cortex. J Neurochem 57:605-614.

Weiss JH, Koh J-Y, Christine CW, Choi DW (1989) Zinc and LTP. Nature 338:67.

Welker E, Soriano E, Van der Loos H (1989) Plasticity in the barrel cortex of the adult mouse: effects of peripheral deprivation on GADimmunoreactivity. Exp Brain Res [Erratum (1989) 77:666-667] 74:441-452.

Westbrook GL, Mayer ML (1987) Micromolar concentrations of Zn2+ antagonize NMDA and GABA responses of hippocampal neurons. Nature 328:640-643.

Woolsey TA, Van der Loos H (1970) The structural organization of layer IV in the somatosensory region (SI) of mouse cerebral cortex. The description of a cortical field composed of discrete cytoarchitectonic units. Brain Res 17:205-242.

Xie X, Smart TG (1994) Modulation of long-term potentiation in rat hippocampal pyramidal neurons by zinc. Pflügers Arch 427:481-486.

Zheng F, Gingrich MB, Traynelis SF, Conn PJ (1998) Tyrosine kinase potentiates NMDA receptor currents by reducing tonic zinc inhibition. Nat Neurosci 3:185-191. 